\title{
Status of Prevention of Mother-to-Child Transmission (PMTCT) Services Utilization and Factors Affecting PMTCT Service Uptake by Pregnant Women Attending Antenatal Care Clinic in Selected Health Facilities of Afar Regional State, Ethiopia
}

\author{
Chalachew Genet Akal (iD) ${ }^{1}$ and Dessie Tegegne Afework ${ }^{2}$ \\ ${ }^{1}$ MSc in Medical Microbiology, College of Medicine and Health Science, Bahir Dar University, Bahir Dar, Ethiopia \\ ${ }^{2}$ MSc in Medical Microbiology, Department of Medical Laboratory Science, Debre Tabor University, Debre Tabor, Ethiopia \\ Correspondence should be addressed to Chalachew Genet Akal; chaliegenet@gmail.com
}

Received 9 July 2018; Accepted 13 November 2018; Published 12 December 2018

Academic Editor: Amir Radfar

Copyright (C) 2018 Chalachew Genet Akal and Dessie Tegegne Afework. This is an open access article distributed under the Creative Commons Attribution License, which permits unrestricted use, distribution, and reproduction in any medium, provided the original work is properly cited.

\begin{abstract}
Mother-to-child transmission (MTCT) is the predominant way for children to acquire human immunodeficiency virus (HIV) infection worldwide including Ethiopia. Thus, objective of this study was to determine the status of prevention of mother-to-child transmission (PMTCT) services utilization and factors affecting PMTCT utilization in health facilities of Afar region, Ethiopia. A cross-sectional study was conducted from December 2014 to April 2015 taking 347 pregnant women and 22 health care providers. Data were collected using a questioner prepared separately for pregnant women and health care providers involved in PMTCT service delivery. Data were analyzed using SPSS considering $P$ value $<0.05$ statistical significant. The study indicated that the PMTCT service utilization was $67.7 \%$. The study also showed that there is statistically significant association in using PMTCT service with women education level, monthly income, and residence around PMTCT site. Though not statistically significant, excess waiting time, limited physical access to PMTCT sites, and transportation problem were identified as barriers for PMTCT service utilization by pregnant women. Though knowledge of mothers on MTCT of HIV and PMTCT service utilization in agropostural community of Afar region was promising, there were also different barriers identified hindering PMTCT utilization. Thus, we recommend "Wored" and zonal health office to create awareness on significance of PMTCT service in the community, enhance accessibility of PMTCT sites, provide up-to-date trainings for health care providers, and ensure constant supply for PMTCT service.
\end{abstract}

\section{Introduction}

Women living with human immunodeficiency virus (HIV) infection can give birth to infants infected with HIV which is known as mother-to-child transmission (MTCT) contributing major proportion for new HIV infections among children. Mother-to-child transmission of HIV is causing significant impact contributing 700,000 estimated new HIV infections in children annually in the globally [1]. In 2016, there were 1.8 million children living with HIV globally where $90 \%$ of the infections were acquired through MTCT of HIV [2]. Based on 2016 World Health Organization
(WHO) data, around $76 \%$ of all pregnant women living with HIV globally received medicines that prevent HIV transmission to their babies [3]. WHO recommends countries, including Ethiopia, to implement prevention of mother-to-child transmission (PMTCT) as it is the most effective strategy for preventing HIV to the pediatrics [4]. In Ethiopia, even though the number of sites and PMTCT coverage vary among region, the average PMTCT service coverage was $54.9 \%$ in $2012 / 13$. Moreover, the national coverage of antiretroviral therapy for HIV-positive pregnant mothers in 2012 was $42.9 \%$, and the Afar regional state coverage (the present study site) was only $20 \%$ in the same 
year [5]. Without preventive interventions, more than a third of the infants born to HIV-positive mothers will contract HIV [6].

Different countries, including Ethiopia, are providing PMTCT services in different health facilities as part of antenatal care (ANC). Despite an increase in the number of health facilities (HFs) providing PMTCT services in Ethiopia, the proportion of HIV-positive pregnant women who receive antiretroviral (ARV) drugs for PMTCT remains low. In 2009, only 8 percent of HIV-positive pregnant women received antiretroviral prophylaxis. As a result, many women end up grappling with their HIV status unaided and alone $[5,7]$. Moreover, Ethiopia is implementing universal HIV screening of pregnant women since 2007 and working to achieve 90-90-90 treatment target endorsed in the 2016 United Nations political declaration on ending AIDS. Moreover, Ethiopia is also working to eliminate MTCT of HIV by 2020 [2].

As being reported by different studies, different factors influence utilization of PMTCT services directly or indirectly: physical accessibility, inconsistent supply of resources for the service, testing methods, lack of trust on result confidentiality, fear of stigma and discrimination, treatment for mothers, feasibility of replacement feeding, inadequate counseling rooms, extent of male involvement in the decision of HIV testing and ANC clinics follow up, lack of information about the presence of the service, economical status, educational level of women, lack of awareness on the significance of voluntary counseling and testing (VCT) and PMTCT, shortage of qualified health professionals, lack of up-to-date training for health care providers, high health care provider turnover, and insufficient allocation of budget [8-10].

Identifying utilization of PMTCT and barriers affecting the utilization of PMTCT service will allow to identify the gap and act accordingly so as to make the service accessible to all pregnant women who need PMTCT service. But up to our knowledge, there is no study done to assess utilization of PMTCT service and associated factors acting as a barrier for the service. Thus the objective of the present study is to determine utilization of PMTCT services and associated factors among pregnant women attending ANC clinic in selected health facilities of Afar region, Ethiopia.

\section{Methods and Materials}

A cross-sectional study was conducted from December 2014 to April 2015 in the Afar regional state of Ethiopia. The Afar regional state is one of the 9 national regional states and two city administrations comprising the Federal Democratic Republic of Ethiopia. The region has a total population of $1,411,092$ accounting $1.7 \%$ of the total Ethiopian population [11]. The region has 3 hospitals, 23 health centers, and 238 health posts as well as 7 health facilities providing PMTCT service as part of ANC services at the time of study. The present study was conducted in randomly selected 5 governmental health facilities (Dubti Hospital, Aysaita District Hospital, Samara Health center, Logia health center, and Chifra Health Center) providing
PMTCT service based on provider-initiated HIV testing and counseling approaches.

From the five health facilities, 347 pregnant mothers attending ANC service were included in the study which was determined using a single proportion formula and by considering design effect of 2 and 10\% nonresponse rate assumption. In addition to 347 pregnant women attending ANC service, all 22 health professionals in 5 selected health facilities providing PMTCT service as part of ANC were included in the study to identify factors affecting PMTCT service utilization. The 347 study participants (pregnant women) were proportionally allocated for 5 health facilities providing PMTCT service based on the total population they serve (i.e., the highest study participants were recruited from the health facility serving the highest population). After proportional allocation, convenient sampling technique was used for each health facility until the required number is reached. Then, the data were collected both from selected pregnant women visiting ANC and health professionals using the pretested questioner which was developed in English and translated to the local language (Amharic). Before data collection started, the "Amharic" questioner was back translated into English for validation. Ten trained data collectors who have diploma in health science were involved in data collection through face-to-face interview supervised by three supervisors after obtaining ethical clearance from Samara University and permission letter from Afar Regional Health Bureau. The questioner was designed to collect data on sociodemographic, acceptability of PMTCT service by local community, and associated factors in the utilization of PMTCT service.

To maintain data quality, the questioner was pretested in Awash Arba health center which was not part of the present study. Moreover, every questioner was checked by data collectors and supervisors for inconsistency and incompleteness at the end of data collection. The collected data were analyzed using SPSS for different descriptive statistics, and associations were assessed by using the $x^{2}$ test. The bivariant logistic regression analyses were used to see the relation between dependent variable and independent variables considering a $P$ value $<0.05$ as statistically significant. Finally, the adjusted odds ratio was used to see the strongest predictors and avoid the confounders.

\section{Results}

From 347 pregnant women included in the study, 34.9\% were $19-25$ years old and $57.1 \%$ of were from hospitals. Moreover, $72.9 \%$ and $54.6 \%$ of pregnant women included were from urban and have monthly income less than 1000 Ethiopian birr (ETB), respectively. Furthermore, the study also showed that $89.3 \%, 77.2 \%, 35.7 \%$, and $50.1 \%$ pregnant women participated in the study were married, Muslims, illiterate, and housewives, respectively (Table 1 ).

The study indicated that 22 health professionals were providing PMTCT services (Figure 1) with a mean age of 26.4 years. Among 22 health professionals, 16 of them were females. The health professionals in each three health 
TABLE 1: Sociodemographic characteristics of the pregnant women attending ANC service in selected health facilities of Afar region, Ethiopia, from December 2014 to April 2015.

\begin{tabular}{|c|c|c|c|}
\hline $\begin{array}{l}\text { Sociodemographic } \\
\text { variables }\end{array}$ & Categories & Frequency & Percent \\
\hline \multirow{6}{*}{ Age group $(n=347)$} & $<18$ years old & 49 & 14.1 \\
\hline & $19-25$ years old & 121 & 34.9 \\
\hline & 26-30 years old & 100 & 28.8 \\
\hline & $31-35$ years old & 60 & 17.3 \\
\hline & $36-40$ years old & 9 & 2.6 \\
\hline & $>40$ years old & 8 & 2.3 \\
\hline \multirow{3}{*}{ Marital status $(n=347)$} & Unmarried & 9 & 2.6 \\
\hline & Married & 310 & 89.3 \\
\hline & Divorced & 28 & 8.1 \\
\hline \multirow{4}{*}{$\begin{array}{l}\text { Educational status }(n= \\
\text { 347) }\end{array}$} & $\begin{array}{c}\text { Cannot read and } \\
\text { write }\end{array}$ & 124 & 35.7 \\
\hline & $1-4$ (1st cycle) & 97 & 28.0 \\
\hline & $5-8$ (2nd cycle $)$ & 97 & 28.0 \\
\hline & $\begin{array}{c}\text { High school and } \\
\text { above }\end{array}$ & 29 & 8.4 \\
\hline \multirow{2}{*}{ Residence $(n=347)$} & Urban & 253 & 72.9 \\
\hline & Rural & 94 & 27.1 \\
\hline \multirow{3}{*}{ Religion $(n=347)$} & Orthodox & 68 & 19.6 \\
\hline & Muslim & 268 & 77.2 \\
\hline & Others & 11 & 3.2 \\
\hline \multirow{5}{*}{ Ethnicity $(n=347)$} & Afar & 254 & 73.20 \\
\hline & Amhara & 42 & 12.10 \\
\hline & Tigre & 35 & 10.08 \\
\hline & Oromo & 10 & 10.08 \\
\hline & Others & 6 & 1.72 \\
\hline \multirow{5}{*}{ Address $(n=347)$} & Dubti hospital & 129 & 37.2 \\
\hline & $\begin{array}{l}\text { Aysaita District } \\
\text { Hospital }\end{array}$ & 69 & 19.9 \\
\hline & $\begin{array}{l}\text { Logia health } \\
\text { center }\end{array}$ & 66 & 19.0 \\
\hline & $\begin{array}{l}\text { Samara Health } \\
\text { center }\end{array}$ & 47 & 13.5 \\
\hline & $\begin{array}{l}\text { Chifra Health } \\
\text { Center }\end{array}$ & 36 & 10.4 \\
\hline \multirow{5}{*}{$\begin{array}{l}\text { Monthly income in birr } \\
(n=347)\end{array}$} & $<500$ birr & 75 & 21.6 \\
\hline & 500-1000 birr & 113 & 32.6 \\
\hline & $1000-1500$ birr & 53 & 15.3 \\
\hline & $>1500$ birr & 54 & 15.6 \\
\hline & I do not know & 52 & 15.0 \\
\hline
\end{tabular}

facilities (Chifra Health Center, Logia health center, and Aysaita hospital) were 4. The rest Dupti hospital and Samara Health center have 7 and 3 health professionals, respectively. In the study, 16 of the PMTCT providers had taken PMTCT training at least once in their life time.

Time of ANC follow up initiation varied among pregnant women from 14 to 16 weeks by $59.7 \%$ to $24-28$ weeks by $17 \%$, respectively. The decision to start ANC service was made by themselves, by husband/partner involvement, and health professional involvement in $57.6 \%, 36.3 \%$, and $6.1 \%$, respectively. Being asked about why they attended ANC service, $32.46 \%, 22.2 \%, 18.7 \%, 6.3 \%$, and $4.9 \%$ of pregnant women replied that they attended ANC to know the status of their pregnancy and their fetus health, to get vaccine, to get treatment for their disease, and to get family planning



FIgUre 1: Professional mix of health professionals providing PMTCT service in five health facilities studied from December 2014 to April 2015 in Afar regional state, Ethiopia.

service after delivery as well as to test for HIV so as to use PMTCT service, respectively. The study also showed that $46.4 \%$ of the study participants have knowledge about MTCT of HIV.

In the study, $246(70.9 \%)$ pregnant women were tested for HIV where $81.3 \%, 13.8 \%$, and $4.9 \%$ of them were negative, positive, and did not know their status, respectively. Among HIV-positive pregnant women, 23 of them took ART drugs and prophylaxis for their babies making utilization of PMTCT service in the study area $67.7 \%$ (Figure 2). Moreover, pregnant women asked on their baby feeding habit/plan, and all preferred to feed breast milk than formula feeding irrespective of their HIV status.

Being asked about their reason why they were not voluntary to be tested for HIV during their ANC follow up, $34(33.7 \%)$ of them reported for the lack of awareness and knowledge followed by doubt on result confidentiality (24.7\%), absence and carelessness of health professionals providing PMTCT service (21.8\%), lack of interest (9.8\%), tested before (7\%), and fear of stigma (3\%). Most of the pregnant women (46.4\%) attending ANC follow up believed that HIV can be transmitted from mother to their child. But, $29.4 \%$ believed that it cannot be transmitted and $24.2 \%$ did not know any information. Among pregnant women who believed HIV transmission from mother to new born, 33.5\% reported that the transmission is in three ways (during pregnancy, during delivery, and through breast feeding), while $31.1 \%, 24.2 \%$, and $11.2 \%$ believed only through delivery, during pregnancy, and through breast feeding, respectively.

The study showed that there were association of PMTCT service utilization with women education, having monthly income $\geq 1000 \mathrm{ETB}$, residence around PMTCT service site than those who cannot read and write, have monthly income $<1000 \mathrm{ETB}$, and live far from PMTCT sites, respectively. Moreover, the association was significant with the $P$ values $0.001,0.001$, and 0.001 , respectively (Table 2 ).

Different factors which prevent pregnant women from using PMTCT service utilization were identified by pregnant women attending ANC. These include excess waiting time in 


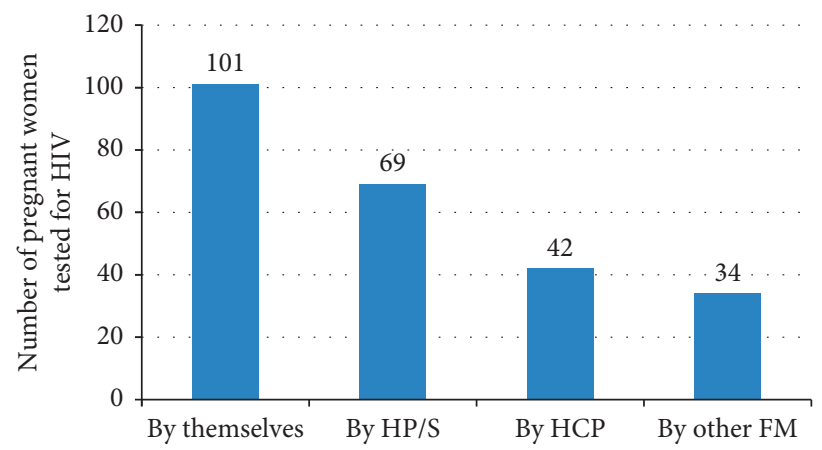

FIGURE 2: The involvement of different parties in the decision of pregnant women for HIV testing during their ANC follow up in selected health facilities of Afar region, Ethiopia from December 2014 to April 2015. HP/S: husband or partner support; HCP: health care provider; FM: family members.

TABLE 2: Association of sociodemographic and other possible potential risk factors with PMTCT service utilization in relation to ANC service in selected health facilities of Afar region, Ethiopia, from December 2014 to April 2015.

\begin{tabular}{|c|c|c|c|c|c|}
\hline \multirow{2}{*}{ Variables } & \multirow{2}{*}{ Categories } & \multicolumn{2}{|c|}{$\begin{array}{l}\text { PMTCT utilization } \\
\text { related to ANC service }\end{array}$} & \multirow{2}{*}{ COR (95\% CI); $P$ value } & \multirow{2}{*}{ AOR $(95 \% \mathrm{CI}) ; P$ value } \\
\hline & & Utilized & $\begin{array}{c}\text { Not } \\
\text { utilized }\end{array}$ & & \\
\hline \multirow{2}{*}{$\begin{array}{l}\text { Residence near PMTCT service } \\
\text { site }\end{array}$} & Yes $(\leq 1 \mathrm{~km})$ & $244(70.3 \%)$ & $28(8.07 \%)$ & 2.5 (1.25-3.9); 0.001 & $6.5(2.35-18.40) ; 0.001$ \\
\hline & No & $\begin{array}{c}106 \\
(30.54 \%)\end{array}$ & $25(7.20 \%)$ & 1 & 1 \\
\hline \multirow{2}{*}{ Educational status } & Could read and write & $\begin{array}{c}208 \\
(59.94 \%)\end{array}$ & $15(4.32 \%)$ & $3.4(2.3-7.46) ; 0.001$ & $11.3(2.8-15) ; 0.001$ \\
\hline & Could not read and write & $97(27.95 \%)$ & $27(7.78 \%)$ & 1 & 1 \\
\hline \multirow[b]{2}{*}{ Knowledge about PMTCT service } & Yes & $177(51 \%)$ & $13(3.74 \%)$ & 3.09 (1.54-6.2); 0.001 & $0.9(0.8-5.25) ; 0.08$ \\
\hline & No & $\begin{array}{c}128 \\
(39.90 \%)\end{array}$ & $29(8.35 \%)$ & 1 & 1 \\
\hline \multirow{2}{*}{ Income level per month } & $\geq 1000 \mathrm{ETB}$ & $\begin{array}{c}139 \\
(40.05 \%)\end{array}$ & $20(5.76 \%)$ & $3.4(1.56-8.04) ; 0.001$ & $5.6(2.05-15.03) ; 0.001$ \\
\hline & $<1000 \mathrm{ETB}$ & $\begin{array}{c}166 \\
(47.53 \%)\end{array}$ & $22(6.32 \%)$ & 1 & 1 \\
\hline \multirow{2}{*}{ Access for the PMTCT service site } & Yes $(\leq 1 \mathrm{~km})$ & $244(70.3 \%)$ & $28(8.07 \%)$ & 2.5 (1.25-3.9); 0.001 & 6.5 (2.35-18.40); 0.001 \\
\hline & No $(>1 \mathrm{~km})$ & $61(17.58 \%)$ & $14(4.03 \%)$ & - & 1 \\
\hline
\end{tabular}

$\mathrm{AOR}=$ adjusted odds ratio; $\mathrm{COR}=$ crude odds ratio; 1 = reference value; $\mathrm{PMTCT}=$ prevention of mother-to-child transmission.

uncomfortable hot waiting rooms, limited physical access to health facilities, and lack of transportation system. But, the association was not significant with the $P$ values of $0.32,0.22$, and 0.44 , respectively.

Moreover, PMTCT providers were also asked on the list of 10 potential barriers identified from literatures either the barriers listed are a challenge or not in their health facility in utilization of PMTCT service. PMTCT providers were asked to respond by saying as not a challenge, simple challenge, or major challenge. Among the potential factors listed, educational level and time shortage of PMTCT service users were not considered as a major challenge by all PMTCT providers in their health facility. But lack of up-to-date training following PMTCT guideline modification was considered as a major challenge by $14(63.6 \%)$ service providers (Figure 3 ).

\section{Discussion}

The World Health Organization identified 22 priority countries with the highest PMTCT service need. Among these, the top 10 countries including Ethiopia account $75 \%$ from the total global PMTCT service need. It was estimated that the effective scaling up of PMTCT interventions in these countries would prevent over 250,000 new infections annually [12].

The knowledge of pregnant women included in the present study about MTCT of HIV was quite higher (46.4\%) than the Ethiopian Demographic and Health Survey (EDHS) data of the region in 2014 (36\%). This variation might be explained that the present study was conducted in urban and semiurban community compare to the Ethiopian Demographic and Health Survey (EDHS) data conducted in the total Afar population. This result, however, was lower than a similar study conducted in Addis Ababa, 90.3\% [8], and Sebeta town, Ethiopia (64.9\%) [13]. This variation might be due to the difference in education level where $35.7 \%$ of the study participants in the present study were illiterate which is higher than those in the study in Addis Ababa (21\%) [8] and Sebeta town (17.1\%) [13]. Moreover, there was a difference in 


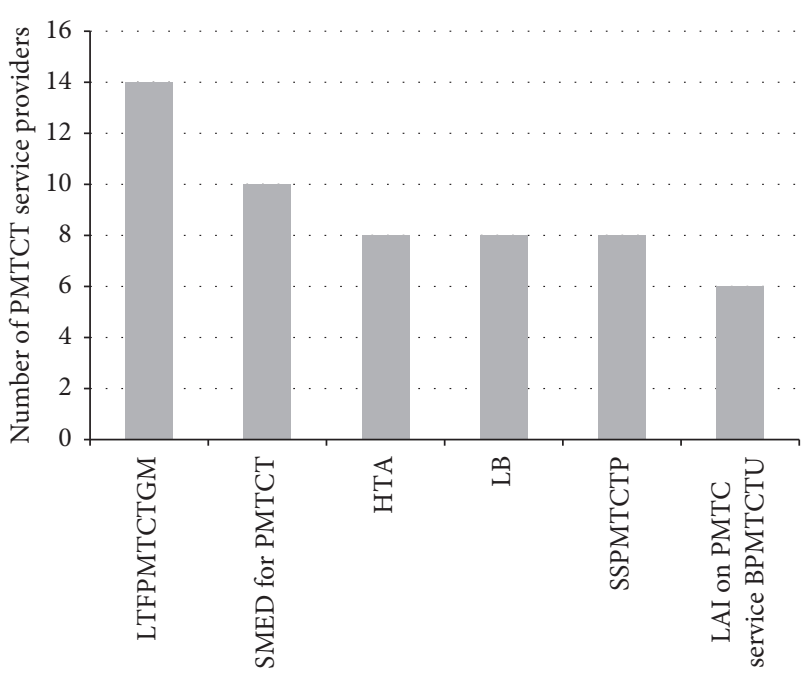

FIgURE 3: Factors listed by health professionals as a major challenge in utilization of PMTCT service in five health facilities studied from December 2014 to April 2015 in the Afar regional state, Ethiopia. LTFPMTCTGM: lack of training following PMTCT guideline modification; SMED for PMTCT: shortage of materials, equipment, and drug for PMTCT, HAT: hot temperature of the area; LB: language barrier; SSPMTCTP shortage of skilled PMTCT providers; LAI on PMTC service BPMTCTU: lack of awareness and ignorance on PMTCT service by PMTCT users.

urbanization between the country capital Addis Ababa and agropastoral community of the present study area.

From the total pregnant women studied, 246 (70.9\%) were tested for HIV. This was lower than a study conducted in Addis Ababa [8], Jinka towns [14], Sebeta town [13], and Horo Guduru Wollega zone [10] of Ethiopia where 94\%, $96.4 \%, 86.9 \%$, and $83.8 \%$ got tested for HIV, respectively. This might be due to the living style (agropastoral) and educational status difference among the population studied as well as study time gap. The present study also showed that $28 \%$ of pregnant women tested for HIV were by their husband/partner support. Moreover, the prevalence of HIV in the present study was $13.8 \%$. This was higher than from EDHS data on HIV prevalence in Afar region (9\%) [11] and studies conducted in Jinka town [14] and Horo Guduru Wollega zone [10] which were $3.4 \%$ and $1.3 \%$, respectively. This can be explained that the present study area is located in the main import-export road corridor of Ethiopia which runs from Addis Ababa to Djibouti hosting many track drivers.

Though PMTCT service is known to reduce the transmission of HIV from mother to child, its use has been limited because of various barriers. The present study had revealed different barriers that might hinder the success of the PMTCT program in both health centers and hospitals. The barriers identified in the present study by pregnant women as a challenge to use PMTCT service were lack of awareness and information access, being uneducated, physical inaccessibility of PMTCT service sites, low income, low involvement of male partners, fear of discrimination and stigma by the community, and financial and transportation problems. This finding agreed with a study in Addis Ababa (Ethiopia) which identified inconsistent supplies, inadequate counseling rooms, limited access to PMTCT service information, HIV/AIDS-related stigma and discrimination in the community, low male involvement, inadequate knowledge, inaccessibility of PMTCT services (unpublished MSC thesis). Moreover, the barriers identified in the present study finding also aggress with a study done in the Horo Guduru Wollega zone of Ethiopia which reported residence, mother's educational status, and male partner involvement during ANC [10].

Moreover, PMTCT service providers in the present study identified lack of trainings following PMTCT guideline modification, hot temperature of the area, shortage of skilled PMTCT provider, lack of awareness of PMTCT service users, and lack of materials and equipment as a major challenge to use PMTCT service. Similar reports on some factors were also identified in the study done in Cameroon as a challenge in using the service which includes low level of education and level of male involvement [15]. Furthermore, the present study goes in line with the study conducted in Addis Ababa, Ethiopia, which reported shortage of PMTCT-trained staff, physical inaccessibility of health facilities, inadequate maternal health services, lack of separate and adequate space for PMTCT services, and economic factors as a major barrier for using PMTCT service [8].

\section{Limitation}

Since the study is conducted on those pregnant mothers attending their ANC follow up in the health facility, the study result cannot reflect the situation in the whole Afar regional state since there are mothers who do not seek ANC follow up. The sampling technique used in the present study was convenient sampling technique which will not give equal chance to be included in the study for all pregnant women using the health facility. Since $35.7 \%$ of our study participants were illiterate, there may be a recall bias which will intern affect the result the study. Furthermore, the present study is a cross-sectional study, and causality cannot be established between the independent and dependent variables.

\section{Conclusion}

Overall, the present study showed promising findings on knowledge of mothers on MTCT of HIV and level of PMTCT service utilization. On the contrary, there were also different major barriers identified in PMTCT service utilization by pregnant women and PMTCT service providers working ANC. These major barriers identified by pregnant women in using PMTCT service include being uneducated, physical inaccessibility, and limited knowledge of PMTCT service sites. Moreover, lack of up-to-date PMTCT training, lack of materials and equipment for PMTCT service, hot temperature of the area, language barrier, and low number of PMTCT providers were the major barriers identified by the PMTCT service provider in providing PMTCT service.

Thus, to improve the accessibility and utilization of PMTCT service, major investment is required by the 
regional and federal government of Ethiopia to minimize major barriers identified by both service users and providers. These can be done thorough expansion of formal education, construction of roads leading to PMTCT sites, expansion of PMTCT sites, and enhancing the overall economic status of the community. On the contrary, we recommend the local and zonal health office to perform activities which require low financial investment like creating awareness on the significance of PMTCT service in the community and role of husband in PMTCT service through health education, providing up-to-date trainings for PMTCT service providers, ensuring constant supply of materials and reagents for PMTCT service.

Moreover, heath education by health extension workers (HEWs) must be given for pregnant women and to the community at large so as to reduce the HIV positivity in pregnant women identified in the study. Moreover, we also recommended HEWs to provide health education for pregnant women to deliver in health facilities than at home with traditional birth attendant (TBA) involvement. Finally, we recommend similar study to be conducted covering the large area of agropostural community of the Afar regional state of Ethiopia to get a better picture.

\section{Abbreviation}

AIDS: Acquired immune deficiency syndrome

ANC: Antenatal care

ART: Antiretroviral therapy

EDHS: Ethiopian Demographic and Health Survey

ETB: Ethiopian birr

HEW: Health extension worker

HIV: Human immuno-deficiency virus

MOH: Ministry of Health

MTCT: Mother-to-child transmission

NIH: National Institutes of Health

PMTCT: Prevention of mother-to-child transmission

VCT: Voluntary counseling and test

WHO: World Health Organization.

\section{Data Availability}

The data used to support the findings of this study are available from the corresponding author upon request.

\section{Conflicts of Interest}

The authors declare that they have no conflicts interests.

\section{Authors' Contributions}

Both authors involved in the designing of the study and writing the manuscript. Moreover, both authors also approved the final manuscript for publication.

\section{Acknowledgments}

It is conducted as Part of our academic employment in Samara University. The authors would like to acknowledge the study participants for their participation in the study as the study was ineffective without them. Finally, the authors also thank Samara University and Afar Regional Health Bureau which allow us to conduct the study having good intention to know the magnitude of the problem for action.

\section{References}

[1] United States President's Emergency Plan for AIDS Relief, "Prevention of mother-to-child transmission," 2008, https:// www.pepfar.gov/documents/organization/79663.pdf.

[2] Y. Ejigu and B. Tadesse, "HIV testing during pregnancy for prevention of mother-to-child transmission of HIV in Ethiopia," PLoS One, vol. 13, no. 8, Article ID e0201886, 2018.

[3] WHO, "HIV/AIDS data and statistics," 2017, http://www. who.int/hiv/data/en/.

[4] WHO, Antiretroviral Drugs for Treating Pregnant Women and Preventing HIV Infection in Infants: Towards Universal Access: Recommendations for a Public Health Approach, WHO, Geneva, Switzerland, 2006.

[5] Federal Democratic Republic of Ethiopia, Ministry of Health, "Policy and practice information for action," 2014, https://www. unicef.org/ethiopia/FMOHPolicyPracticeBulletinApril14.pdf.

[6] V. Claire, S. Sara, D. Y. Asfaw, and A. Shibru, Mothers' Support Groups in Ethiopia, USAID, Washington, DC, USA, 2008, https://www.intrahealth.org/sites/ihweb/files/files/media/ mothers-support-groups-in-ethiopia-a-peer-support-modelto-address-the-needs-of-women-living-with-hiv/Mothers\% 20Support\%20Groups.pdf.

[7] USAID, "Ethiopia HIV/AIDS health profile," October 2013, http://www.usaid.gov/our_work/global_health/aids.

[8] W. Deressa, A. Seme, A. Asefa, G. Teshome, F. Enqusellassie et al., "Utilization of PMTCT services and associated factors among pregnant women attending antenatal clinics in Addis Ababa, Ethiopia," BMC Pregnancy and Childbirth, vol. 14, no. 1, p. $328,2014$.

[9] M. Gobena, T. Nigatu, B. Yimam et al., "Utilization of Prevention of Mother to Child transmission (PMTCT) services and factors that affect knowledge and service uptake among pregnant women attending antenatal care in East Hararge Zone of Oromia Reginal State," Ethiopian Journal of Health Development, no. 1, pp. 126-135, 2014.

[10] A. Feyera, B. Megerssa, D. Legesse, and F. Hailemichael, "Prevention of mother to child transmission of HIV/AIDS: service utilization and associated factors among selected public health facilities in Ethiopia," Medical Practice and Reviews, vol. 8, no. 11, pp. 1-13, 2017.

[11] Federal Democratic Republic of Ethiopia, Addis Ababa, Ethiopia, Demographic and Health Survey, Central Statistical Authority (CSA), Ethiopia, 2008.

[12] WHO, AVERT HIV/AIDS, "A comprehensive approach to prevention of mother-to-child transmission (PMTCT) of HIV," 2013, https://www.avert.org/professionals/hiv-programming/ prevention/prevention-mother-child.

[13] H. Merga, K. Woldemichael, and L. Dube, "Utilization of prevention of mother-to-child transmission of HIV service and associated factors among antenatal care attending mothers in Sebeta town, Central Ethiopia," Advanced in Public Health, vol. 2016, Article ID 6250898, 10 pages, 2016.

[14] G. Wanzahun and A. Abraham, "Prevalence of HIV/AIDS and its associated factors among prevention of mother-to- 
child transmission (PMTCT) service users in Jinka town health Institutions, South Omo zone," Science Journal of Public Health, vol. 1, no. 3, pp. 125-130, 2013.

[15] G. Awungafac, P. A. Njukeng, J. A. Ndasi, and L. T. Mbuagbaw, "Prevention of mother-to-child transmission of the Human Immunodeficiency Virus: investigating the uptake and utilization of maternal and child health services in Tiko health district, Cameroon," Pan African Medical Journal, vol. 20, p. 20, 2015. 


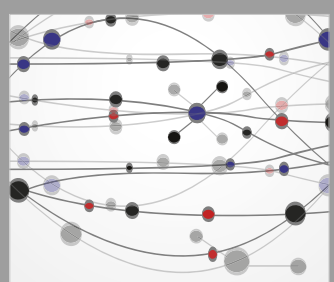

The Scientific World Journal
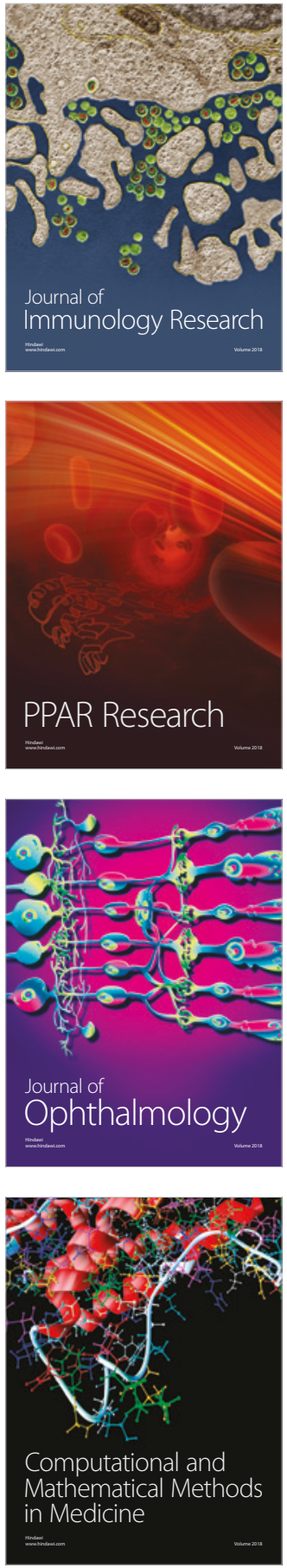



Gastroenterology Research and Practice

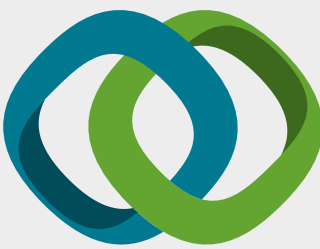

\section{Hindawi}

Submit your manuscripts at

www.hindawi.com


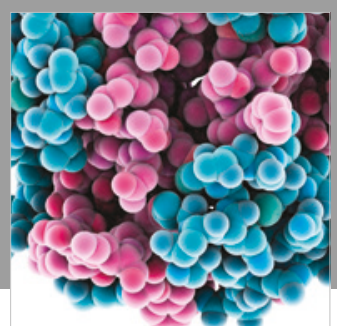

ournal of

Diabetes Research

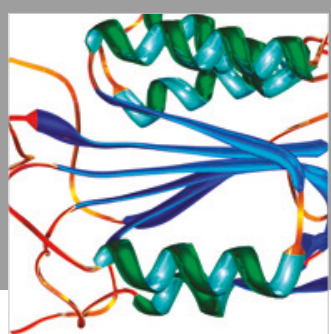

Disease Markers
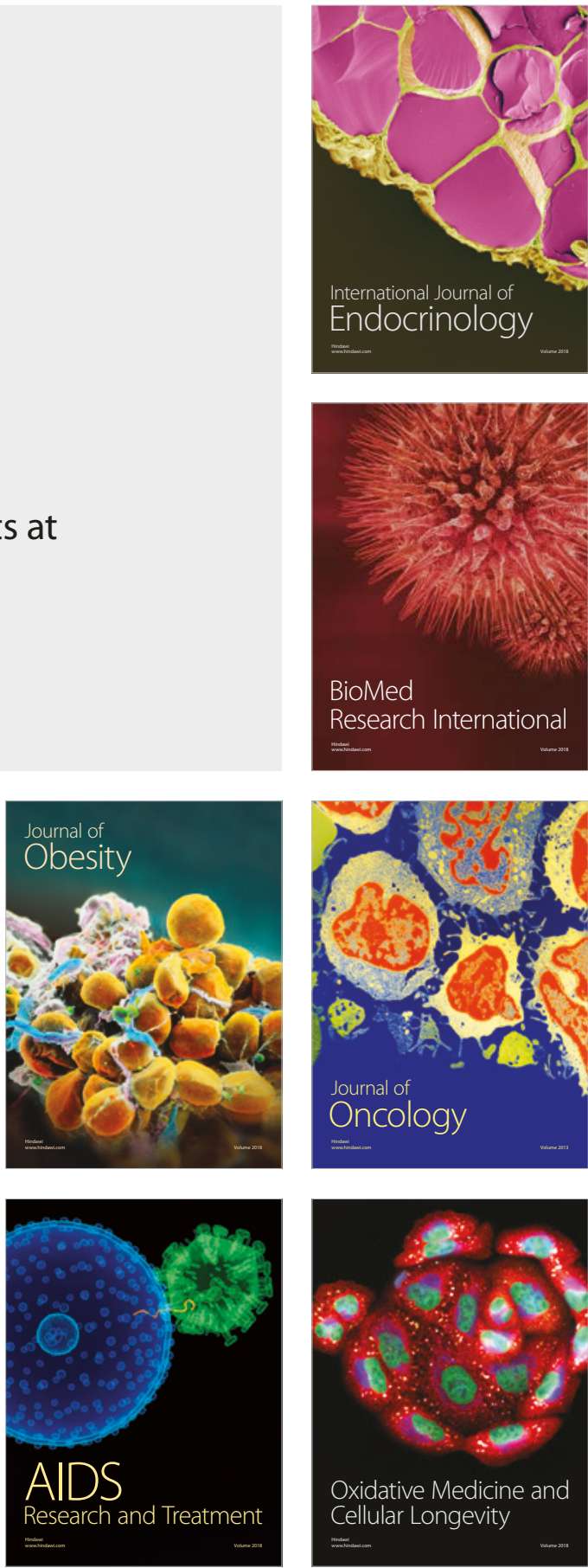